\title{
Nonlinear Dynamics of Blood Pressure Variability After Caffeine Consumption
}

\author{
Theodore G. Papaioannou, PhD; Charalambos Vlachopoulos, MD; Nikolaos loakeimidis, MD; \\ Nikolaos Alexopoulos, MD; and Christodoulos Stefanadis, MD
}

\begin{abstract}
Objectives: The occurrence of continuous blood pressure (BP) fluctuations is an intrinsic feature of the cardiovascular system and is related to complex cardiovascular mechanisms and environmental stimulations or daily habits such as coffee drinking. The aim of this study was to investigate the effect of caffeine on indices expressing the complex and "chaotic" nonlinear characteristics of BP variability.
\end{abstract}

Design: Fourteen healthy subjects consumed $240 \mathrm{mg}$ of caffeine or placebo according to a randomized, double-blind, crossover design. Continuous radial pressure waveforms were recorded by applanation tonometry at baseline and at 30,60,90 and I20 minutes after ingestion of caffeine or placebo.

Methods: Approximate entropy (ApEn) was determined to assess BP irregularity. Detrended fluctuation analysis was also used to quantify the fractal correlation properties of the BP data by estimating the scaling (self-similarity) exponent $\alpha$. Analysis of variance for repeated measurements was used to detect significant changes in the measured variables at 30,60, 90 and 120 minutes after ingestion of caffeine or placebo.

Results: BP fluctuations demonstrated high regularity and predictability as indicated by the low values of ApEn $(0.2 \pm 0.3)$, and they remained unchanged after caffeine ingestion. In contrast, the long-term scaling exponent $\alpha$ of the BP time series was significantly increased from 0.99 to $\mathrm{I} .04 \quad(p=0.0 \mathrm{l})$ after caffeine ingestion, while the placebo induced no significant change.

Conclusions: Caffeine induced greater "randomness" in BP fluctuations, possibly by triggering cardiovascular mechanisms that could not be directly specified but should be investigated in future studies.

Keywords: Applanation tonometry; Approximate entropy; Caffeine; Chaos; Coffee; Detrended fluctuation analysis; Fractal; Nonlinear dynamics; Pressure waveform; Variability

$\mathrm{T}$ he analysis of variability of various biological signals has undergone considerable growth during recent decades. Analysis of linear statistics (e.g., mean values, variability measures and spectra analysis) of biological signals does not directly characterize their complexity, irregularity or predictability. Methods based on non-linear dynamics and "chaos" theories may reveal subtle abnormalities in the cardiovascular regulation mechanisms that may not be uncovered by traditional (i.e., "linear") measures of variability, and thus, they provide a useful tool for in depth evaluation of the properties of complex biological systems.
Cardiovascular research in the field of non-linear dynamics has primarily focused on heart rate and, to a lesser degree, on blood pressure (BP) variability. Several mathematical methods and indices exist for the characterization of the non-linear dynamic behavior of biological systems (e.g., approximate entropy, sample entropy, detrended fluctuation analysis, recurrence analysis, Poincare plots, Lyapunov exponents, correlation and fractal dimensions).

The occurrence of continuous BP fluctuations is an intrinsic feature of the cardiovascular system and relates to complex biological mechanisms and environmental stimulations. Caffeine is considered to be the most widely consumed 
pharmacologic substance in the world, rendering its short- and long-term hemodynamic effects an issue of particular research and clinical interest. To our knowledge, no data exist regarding the acute response of $\mathrm{BP}$ variability indices, especially nonlinear indices, to caffeine consumption. The aim of this study was to investigate the effect of caffeine, a well known pressor-altering and vasoactive pharmacological substance, ${ }^{1}$ on indices expressing the complex and chaotic nonlinear characteristics of BP variability.

\section{Materials and Methods}

Fourteen healthy subjects consumed $240 \mathrm{mg}$ of caffeine or placebo orally according to a randomized, double-blind, crossover design. In all cases, caffeine and placebo were administered at approximately the same time (i.e., 8:00 to 10:00 AM). Continuous radial pressure waveforms were recorded by applanation tonometry. A high fidelity micromanometer-tipped probe was used (Millar Instruments, Houston, TX), which provided accurate recordings of peripheral pressure contours as shown previously by comparison with intra-arterial measurements. ${ }^{2}$ In this study, the recorded pressure waveforms were subject to several quality criteria to control for the appropriateness of:

- Average pulse height (i.e., the average of the heights of all the pulses);

- Pulse height variation (i.e., the amount of variation present in the pulse heights. Less than 3\% was accepted, otherwise applanation was repeated);

- Diastolic variation (i.e., the amount of variation present in the diastolic point of the pulse wave indicating how constant the baseline pressure was during the measurement. Less than 3\% was accepted, otherwise applanation was repeated).

Each recording ( $>30$ seconds) consisted of measurements in the supine position at baseline and at 30, 60, 90 and 120 minutes following ingestion of placebo or caffeine. BP measurements and methods regarding radial tonometry have been described in detail previously. ${ }^{3}$ All measurements were obtained after an overnight fast and were performed in a quiet, air-conditioned room after a 15-minute resting period. The same well-trained operator performed all measurements, thus eliminating inter-observer variability. The study was approved by the ethics committee of our hospital, and all participants gave informed consent before entering the study.

Table 1. Descriptive characteristics of the study population.

\begin{tabular}{ll}
\hline Age (years) & $29.2 \pm 1.4$ \\
Gender (males) & 14 \\
Height $(\mathrm{cm})$ & $176 \pm 1.7$ \\
Weight $(\mathrm{kg})$ & $73.6 \pm 3$ \\
Prior coffee consumption (cups/week) & $17.4 \pm 3.2$ \\
\hline
\end{tabular}

\section{Nonlinear analysis of BP variability} Approximate entropy (ApEn)

ApEn $(m, r)$ of a time series measures the logarithmic frequency with which vectors with $m$ components that are close (i.e., within resolution $r$ ) remain close when increasing the number of vector components by one. Small values of ApEn indicate regularity, and large values imply substantial fluctuations or irregularity in a time series. The input values $m=2$ and $r=20 \%$ of the standard deviation of the BP data sets were used in accordance with previous findings with good statistical validity. ${ }^{4}$

\section{Detrended fluctuation analysis (DFA)}

DFA was used to quantify the fractal correlation properties of the BP data by estimating the scaling (self-similarity) exponent $\alpha$. DFA is a modified root mean square analysis of a random walk. ${ }^{5}$ Values of $\alpha$ between 0.5 and 1 indicate persistent long-range correlations, such that small and large $\mathrm{BP}$ values are more likely to alternate. For $\alpha>1$, correlations exist but not in a power-low form. An $\alpha$ value of 1.5 indicates Brownian noise.

More than 30 continuous BP waveforms were acquired resulting in the recording of $>6000$ pressure values. Both ApEn and $\alpha$ were calculated by analyzing all the acquired BP values.

\section{Statistical analysis}

Analysis of variance (ANOVA) for repeated measurements was performed to detect significant changes in the measured variables after caffeine or placebo consumption. Comparison of the variables' responses between the caffeine and placebo sessions was performed using a 2 [drug] x 5 [time] general linear model, namely (caffeine vs placebo) x (baseline, 30, 60, 90, 120 minutes). The significance of the interaction term between caffeine and placebo was tested. A Bonferroni-corrected Student's t-test for multiple comparisons was used to evaluate changes over time for the placebo and caffeine sessions. A two-tailed, paired Student's t-test was applied to assess differences in baseline values between the two sessions. Data are presented as mean \pm standard error of the mean (SEM). Probability levels $<0.05$ were considered statistically significant.

\section{Results}

Descriptive characteristics of the study population are presented in table 1. The mean values of heart rate and systolic, diastolic, and pulse pressures for the caffeine and placebo groups prior to the ingestion of caffeine or placebo are presented in table 2 .

A time domain analysis of BP indicated a significant increase in systolic BP at 30 minutes $(122.7 \pm 1.4 \mathrm{mmHg}, \mathrm{p}=0.001)$ and 60 minutes $(126.1 \pm 2.0, \mathrm{p}=0.003)$ after caffeine intake compared with baseline values (table 1). Diastolic BP was also increased significantly at 30 minutes $(70.3 \pm 1.3 \mathrm{mmHg}$, $\mathrm{p}=0.008)$ and 60 minutes $(72.3 \pm 1.5 \mathrm{mmHg}, \mathrm{p}<0.001)$ after caffeine intake. Finally, heart rate decreased significantly 30 
Table 2. Values (mean \pm SEM) of heart rate and brachial systolic, diastolic and pulse pressures by group prior to the ingestion of caffeine and placebo (baseline).

\begin{tabular}{lccc}
\hline & Caffeine & Placebo & p-value \\
\hline Heart rate (beats/minute) & $69.3 \pm 3.6$ & $70.1 \pm 3.1$ & 0.601 \\
Systolic pressure $(\mathrm{mmHg})$ & $116.2 \pm 1.7$ & $117.1 \pm 1.9$ & 0.567 \\
Diastolic pressure $(\mathrm{mmHg})$ & $67.6 \pm 1.3$ & $67.6 \pm 0.8$ & 1.000 \\
Pulse pressure $(\mathrm{mmHg})$ & $48.6 \pm 1.5$ & $49.5 \pm 1.9$ & 0.566
\end{tabular}

minutes and 60 minutes after caffeine consumption $(63.1 \pm 3.4$ bpm $[\mathrm{p}=0.001]$ and $63.1 \pm 3.1 \mathrm{bpm}[\mathrm{p}=0.012]$, respectively). The placebo had no significant effect on BP or heart rate.

BP fluctuations demonstrated high regularity and predictability as indicated by the low baseline values of ApEn (approximately $0.2 \pm 0.3$ ), and they remained unchanged 2 hours after ingestion of caffeine or placebo. On the other hand, the long-term scaling exponent $\alpha$ of the BP time series was significantly increased from 0.99 to 1.04 ( $\mathrm{p}=0.01$, observed power $=85 \%$ ) after caffeine ingestion, while the placebo induced no significant change (figure 1). Also, the caffeine effect on the scaling exponent $\alpha$ was significantly different from the respective effect of the placebo, as indicated by the statistically significant level $(\mathrm{p}=0.004$, observed power $91.2 \%)$ of the interaction term of the general linear model.

\section{Discussion}

The development of mathematical techniques based on chaos theory and nonlinear dynamics often provides a greater ability to discern meaningful differences between biological signals obtained at pathophysiologically distinct conditions. This study provides evidence that biomedical signals vary in a complex and irregular way even during "steady-state" conditions. However, irregularity in time series data is often overlooked by time domain indices such as mean values or standard deviations.

In the present study, a significant acute increase in systolic and diastolic BP and a decrease in heart rate were observed after caffeine ingestion. These observations are consistent with those of other studies. ${ }^{1,3,6-8}$

The novel finding of this study is the increase in randomness of BP fluctuations after caffeine consumption, as indicated by the rapid increase in the scaling (self-similarity) exponent $\alpha$. It is known that physiological signals yield values of $\alpha$ close to 1 . This was confirmed by the data obtained at baseline in the BP time series in our study ( $95 \%$ CI 0.952 to 1.046 ). After caffeine consumption, a significant increase in $\alpha(95 \%$ CI 1.00 to 1.10$)$ was observed, which indicates a loss in long-range scaling behavior of the BP time series. In contrast to the significant change in the scaling exponent $\alpha$, the ApEn of the short-term BP time series after caffeine intake was not altered significantly. The exact mechanisms responsible for this paradox remain to be clarified.

A potential physiological underlying mechanism of the regulation of BP variability is arterial baroreceptors. In conscious dogs, Wagner et al found that sinoaortic and cardiopulmonary baroreceptor denervation resulted in a decrease in BP complexity, rendering its variation more predictable. ${ }^{9}$ It could be hypothesized that the observed increase in BP randomness in our study might be due to an indirect effect of caffeine on baroreceptor sensitivity.

Additionally, the effect of caffeine on smooth muscle cells and the chaotic, dynamic behavior of capillary vessels ${ }^{10}$ may also be responsible for the regulation of the non-linear characteristics of BP variability. It should be underscored that caffeine has complex actions on the circulatory system, and the final effects on BP modification and control also depend upon the conditions prevailing at the time of administration, the dose used, and possibly the history of exposure to methylxanthine. Because of the various direct and indirect vascular and neural actions of caffeine, the exact mechanisms responsible for the observations of the present study cannot be definitely ascertained, and thus, they require elucidation in future studies.

To our knowledge, until now there have been no data showing the effects of caffeine consumption on the nonlinear dynamics of BP variability. Thus, the clinical relevance of the findings of the present study could be indirectly extrapolated by previous studies investigating the nonlinear dynamics of heart rate or BP variability.

Previous studies have found that a decreased short-term scaling exponent that is calculated using detrended fluctuation analysis yields important prognostic information about the risk of adverse events in patients with implantable cardioverter-defibrillators. ${ }^{11}$ In postinfarction studies, it has been suggested that the short-term scaling exponent $\left(\alpha_{1}\right)$ is a better predictor of adverse outcomes than conventional heart rate variability measures. ${ }^{12,13}$ Also, decreased values of $\alpha_{1}$ have been attributed to vulnerability to ventricular tachyarrhythmias, ${ }^{14,15}$ arrhythmic death, and nonarrhythmic cardiac death ${ }^{13}$ and have been found to precede the onset of paroxysmal atrial fibrillation episodes. ${ }^{16}$ 
In general, decreased complexity and predictability of heart rate and BP fluctuations represent a limited transmission of important physiologic information needed to respond to a changing environment. On the other hand, increased chaos is also related to pathological phenomena. Fibrillations seem to be highly chaotic in nature with a high fractal dimension. ${ }^{17}$

It is not clear whether the observed acute increase in BP randomness represents an unfavorable or beneficial effect. However, although seemingly odd, if rapid responses are required, the presence of chaos may be an advantage in control systems. This also applies to the cardiovascular system. Of course, chaotic systems seem to be quite unreliable because of their extreme sensitivity to initial conditions. However, an increased sensitivity allows a control mechanism to control the system with very small corrective signals, which means that proper feedback is available. Thus, very small adjustments can have large effects, a fact with particular importance to cardiovascular control systems. Consequently, changing the mode of operation of a system (e.g., heart rate or BP) rapidly and without the expenditure of large amounts of energy is of vital importance. It is believed that the constant feedback and small corrective steps necessary to keep such systems in the correct mode are probably not such a drawback, since the expenditure of energy can be small for chaotic systems. ${ }^{18}$ From this point of view, the observed acute effect of caffeine on nonlinear dynamic properties of BP fluctuations could be considered beneficial. This view is in line with previous reports of acute improvement in traditional heart rate variability indices of diabetic patients and healthy volunteers. ${ }^{19,20}$

Some limitations of this study should be mentioned. It is known that nonlinear analysis requires long sequences, and thus, a longer recording of BP time series might be even more appropriate for the investigation of the effects of caffeine on the complex characteristics of a BP time series. A heart rate time series that is long enough to allow for nonlinear analysis data would also be useful to better understand the reported effects of caffeine on BP variability. In this study, the small number in the study population could be considered a limitation. To our belief, more studies are required to investigate the nonlinear effects of caffeine on hemodynamics in larger and different populations.

The data presented in this study indicate a significant, acute, "chaotic" consequence of a widely and regularly used pharmacological substance on BP variability. It could be hypothesized, based on previous studies, that this effect might be beneficial. This needs to be substantiated by future studies, especially on a long-term basis. These findings may trigger further investigators to uncover the clinical and pathophysiological significance of the alterations in the nonlinear properties of the BP regulatory system.

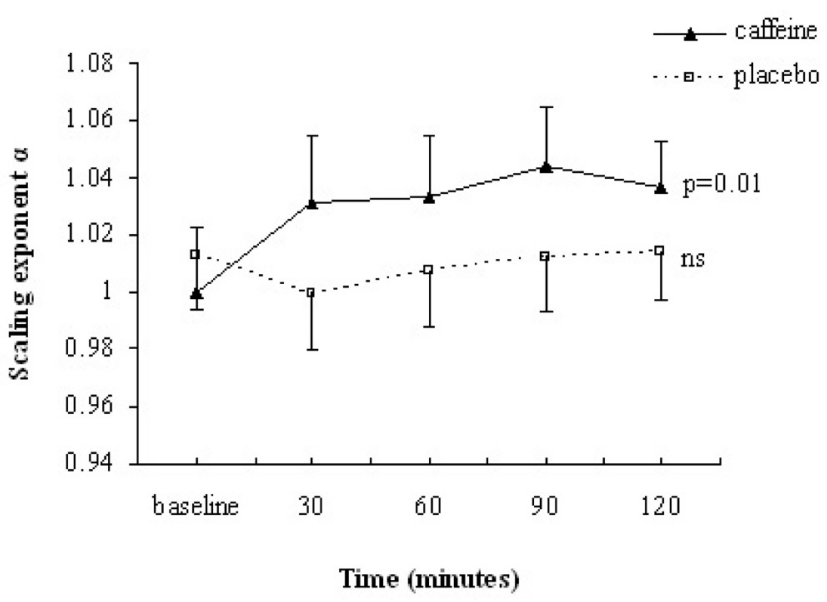

Figure 1. Acute effects of caffeine and placebo on the scaling (self-similarity) exponent $\alpha$. P-values indicate significance of ANOVA for repeated measurements. Bars represent standard errors of the mean values.

\section{References}

1. Papaioannou TG, Karatzi K, Karatzis E, Papamichael C, Lekakis JP. Acute effects of caffeine on arterial stiffness, wave reflections, and central aortic pressures. Am J Hypertens 2005;18:129-136.

2. Kelly R, Hayward C, Ganis J, Daley J, Avolio A, O’Rourke M. Non-invasive registration of the arterial pulse waveform using high-fidelity applanation tonometry. J Vasc Med Biol 1989;1:142-149.

3. Vlachopoulos C, Hirata K, O'Rourke MF. Pressure-altering agents affect central aortic pressures more than is apparent from upper limb measurements in hypertensive patients: the role of arterial wave reflections. Hypertension 2001;38:14561460.

4. Pincus SM, Goldberger AL. Physiological time-series analysis: what does regularity quantify? Am J Physiol 1994;266:H1643-H1656.

5. Peng CK, Havlin S, Stanley HE, Goldberger AL. Quantification of scaling exponents and crossover phenomena in nonstationary heartbeat time series. Chaos 1995;5:82-87.

6. Vlachopoulos C, Hirata K, O'Rourke MF. Effect of caffeine on aortic elastic properties and wave reflection. J Hypertens 2003;21:563-570.

7. Vlachopoulos C, Hirata K, Stefanadis C, Toutouzas P, O’Rourke MF. Caffeine increases aortic stiffness in hypertensive patients. Am J Hypertens 2003;16:63-66.

8. Karatzis E, Papaioannou TG, Aznaouridis K, Karatzi K, Stamatelopoulos K, Zampelas A, Papamichael C, Lekakis J, Mavrikakis M. Acute effects of caffeine on blood pressure and wave reflections in healthy subjects: should we consider monitoring central blood pressure? Int J Cardiol 2005;98:425-430.

9. Wagner CD, Mrowka R, Nafz B, Persson PB. Complexity and "chaos" in blood pressure after baroreceptor denervation of conscious dogs. Am J Physiol 1995;269:H1760-H1766.

10. Tuda I, Tahara T, Iwanaga H. Chaotic pulsation in human capillary vessels and its dependence on mental and physical conditions. Int J Bifurcation and Chaos 1992;2:313-324.

11. Perkiomaki JS, Zareba W, Daubert JP, Couderc JP, Corsello A, Kremer K. Fractal correlation properties of heart rate dynamics and adverse events in patients with implantable cardioverter-defibrillators. Am J Cardiol 2001;88:17-22. 
12. Makikallio TH, Hoiber S, Kober L, Torp-Pedersen C, Peng CK, Goldberger AL, Huikuri HV. Fractal analysis of heart rate dynamics as a predictor of mortality in patients with depressed left ventricular function after acute myocardial infarction. TRACE Investigators. TRAndolapril Cardiac Evaluation. Am J Cardiol 1999;83:836-839.

13. Huikuri HV, Makikallio TH, Peng CK, Goldberger AL, Hintze U, Moller M. Fractal correlation properties of R-R interval dynamics and mortality in patients with depressed left ventricular function after an acute myocardial infarction. Circulation 2000;101:47-53.

14. Makikallio TH, Seppanen T, Airaksinen KE, Koistinen J, Tulppo MP, Peng CK, Goldberger AL, Huikuri HV. Dynamic analysis of heart rate may predict subsequent ventricular tachycardia after myocardial infarction. Am J Cardiol 1997;80:779-783.

15. Makikallio TH, Koistinen J, Jordaens L, Tulppo MP, Wood N, Golosarsky B, Peng CK, Goldberger AL, Huikuri HV. Heart rate dynamics before spontaneous onset of ventricular fibrillation in patients with healed myocardial infarcts. Am J Cardiol 1999;83:880-884.

16. Vikman S, Makikallio TH, Yli-Mayry S, Pikkujamsa S, Koivisto AM, Reinikainen P, Airaksinen KE, Huikuri HV. Altered complexity and correlation properties of R-R interval dynamics before the spontaneous onset of paroxysmal atrial fibrillation. Circulation 1999;100:2079-2084.

17. Garfinkel A, Spano ML, Ditto WL, Weiss JN. Controlling cardiac chaos. Science 1992;257:1230-1235.

18. Lipsitz LA. Age-related changes in the "complexity" of cardiovascular dynamics: A potential marker of vulnerability to disease. Chaos 1995;5:102-109.

19. Richardson T, Rozkovec A, Thomas P, Ryder J, Meckes C, Kerr D. Influence of caffeine on heart rate variability in patients with long-standing type 1 diabetes. Diabetes Care 2004;27:1127-1131.

20. Yeragani VK, Krishnan S, Engels HJ, Gretebeck R. Effects of caffeine on linear and nonlinear measures of heart rate variability before and after exercise. Depress Anxiety 2005;21:130-134.

\section{Author Affiliations}

Theodore G. Papaioannou, PhD, Unit of Biomedical Engineering, First Department of Cardiology, Hippocration Hospital, Medical School, National and Kapodistrian University of Athens, Greece

Charalambos Vlachopoulos, MD, Unit of Biomedical Engineering, First Department of Cardiology, Hippocration Hospital, Medical School, National and Kapodistrian University of Athens, Greece

Nikolaos Ioakeimidis, MD, Unit of Biomedical Engineering, First Department of Cardiology, Hippocration Hospital, Medical School, National and Kapodistrian University of Athens, Greece

Nikolaos Alexopoulos, MD, Unit of Biomedical Engineering, First Department of Cardiology, Hippocration Hospital, Medical School, National and Kapodistrian University of Athens, Greece
Christodoulos Stefanadis, MD, Unit of Biomedical Engineering, First Department of Cardiology, Hippocration Hospital, Medical School, National and Kapodistrian University of Athens, Greece 